\title{
A Mitophagy-Related Gene Signature Associated With Prognosis and Immune Microenvironment in Colorectal Cancer
}

\section{Cong Zhang}

Hospital of Chengdu University of Traditional Chinese Medicine

\section{Cailing Zeng}

Hospital of Chengdu University of Traditional Chinese Medicine

\section{Shaoquan Xiong}

Hospital of Chengdu University of Traditional Chinese Medicine

Zewei Zhao

Chengdu University of Traditional Chinese Medicine

Guoyu Wu ( $\nabla$ wuguoyu_1@163.com )

Hospital of Chengdu University of Traditional Chinese Medicine

\section{Research Article}

Keywords: Colorectal cancer, mitophagy-related genes, signature, prognostic, tumor immune microenvironment

Posted Date: January 4th, 2022

DOI: https://doi.org/10.21203/rs.3.rs-1157954/v1

License: (c) (i) This work is licensed under a Creative Commons Attribution 4.0 International License. Read Full License 


\section{Abstract}

Background: Colorectal cancer (CRC) is a heterogeneous disease and one of the most common malignancies in the world. Previous studies have found that mitophagy plays an important role in the progression of colorectal cancer. This study is aimed to investigate the relationship between mitophagyrelated genes and the prognosis of patients with CRC.

Methods: Gene expression profiles and clinical information of CRC patients were obtained from The Cancer Genome Atlas (TCGA) and Gene Expression Omnibus (GEO) databases. Univariate Cox regression and the least absolute shrinkage and selection operator (LASSO) regression analysis were used to establish the prognostic signature composed of mitophagy related genes. Kaplan-Meier curve and receiver operating characteristic $(\mathrm{ROC})$ curve were used to analyze patient survival and verify the predictive accuracy of the signature, respectively. Construction of a nomogram prognostic prediction model was based on risk scores and clinicopathological parameters. Using the Genomics of Drug Sensitivity in Cancer (GDSC) database and Tumor Immune Dysfunction and Exclusion (TIDE) algorithm to estimate the sensitivity of chemotherapy, targeted therapy and immunotherapy.

Results: A total of 44 mitophagy-driven genes connected with CRC survival were identified, and prognostic signature was established based on the expression of 10 of them (AMBRA1, ATG14, MAP1LC3A, MAP1LC3B, OPTN, VDAC1, ATG5, CSNK2A2, MFN1, TOMM22). Patients were divided into high-risk and low-risk groups based on the median risk score, and the survival of patients in the high-risk group was significantly shorter than that of the low-risk group among the TCGA cohort (median OS 67.3 months vs not reached, $\mathrm{p}=0.00059$ ) and two independent cohorts from GEO (median OS in GSE17536: 54.0 months vs not reached, $p=0.0082$; in GSE245: 7.7 months vs not reached, $p=0.025$ ). ROC curve showed that the area under the curves (AUC) of 1-, 3- and 5-year survival were $0.66,0.66$ and 0.64 , respectively. Multivariate Cox regression analysis confirmed the independent prognostic value of the signature. Then we constructed a nomogram combining the risk score, age and $\mathrm{M}$ stage, which had a concordance index of survival prediction of $0.77(95 \% \mathrm{Cl}=0.71-0.83)$ and more robust predictive sensitivity and specificity. Results showed that CD8+ T cells, regulatory $T$ cells and activated NK cells were significantly more abundant in the high-risk group. Furthermore, patients in the high-risk group were more sensitive to potential targeted therapies, including Motesanib, ATRA, Olaparib, Selumetinib, AZD8055 and immunotherapy.

Conclusion: In conclusion, we constructed and validated a novel mitophagy related gene signature that can be used as an independent prognostic biomarker for CRC, and may lead to better stratification and selection of precise treatment for CRC patients.

\section{Introduction}

Colorectal cancer (CRC) is the third most common malignancy in the world and is considered the second leading cause of cancer-related death, causing an estimated more than 800,000 deaths in 2018 [1]. It is a 
heterogeneous disease, which is the result of accumulation of mutations over time under the influence of environmental, genetic and other risk factors [2]. Currently, effective therapies for CRC mainly include laparoscopic surgery for the primary tumor, combined radiation therapy and palliative chemotherapy [3]. It is noteworthy that $25 \%$ of CRC patients have liver metastases at diagnosis, and $50 \%$ will develop long distant metastasis during the course of the disease [4]. Early detection through screening has improve 5yaer survival of CRC patients in developed countries, but the prognosis for metastatic CRC ( $\mathrm{MCRC}$ ) patients remains poor, with a median 5-year survival rate of just $12.5 \%$ in the United States $[5,6]$. Therefore, metastasis and recurrence of CRC seriously affect the prognosis of patients [7]. Although immune checkpoint inhibitors have been reported to have achieved significant efficacy in a cohort of CRC patients [8-10]. However, immune checkpoint inhibitors remain ineffective in mismatch repair-proficient (pMMR), microsatellite stable (MSS) or low levels of microsatellite instability (MSI-L) CRC [11]. Therefore, it is of clinical importance to determine the patients' prognosis and which patients will benefit from immunotherapy.

Mitophagy, a selective autophagy process, is a fundamental mechanism of conservation from yeast to humans, which controls the quality and quantity of mitochondria by degrading dysfunctional mitochondria $[12,13]$. It plays a key role in the survival of cancer cells by influencing the metabolic reprogramming of mitochondria in tumors and the accumulation/elimination rate of damaged mitochondria through different mechanisms to maintain mitochondrial homeostasis [13]. On the other hand, some studies have found mutations or silencing of the mitophagy adaptor in some cancer types, thus demonstrating the suppressive role for mitophagy on tumors [14]. Recently, Chen et al. found that DJ-1 (Parkinson's disease-associated protein 7, PARK7) activated mitophagy can remove dysfunctional mitochondria and inhibit the apoptosis of metastatic colorectal adenocarcinoma cells, thereby promoting the progression of CRC [15]. In addition, previous studies have found that Tanshinone IIA (Tan IIA) regulates mitochondrial homeostasis by modulating mitophagy, thereby exerting suppressive effects on CRC [16]. Although current evidence had suggested the relationship between mitophagy related genes and prognosis in some cancer types, they mainly focused on the function of a single gene.

In this study, Cox proportional hazard regression analysis was used to screen genes associated with mitophagy for prognosis. The resulting genes were then applied to LASSO regression analysis to establish gene signature and validated in two independent cohorts from GEO (GSE17536 and GSE24551). Patients were divided into high and low risk groups based on the median risk score and survival analyses were performed to assess the prognostic value of the signature. Gene ontology (GO), Kyoto Encyclopedia of Genes and Genomes (KEGG) and Gene Set Enrichment Analysis (GSEA) were used to analyze differences in signaling pathways in high and low risk groups. After that, a nomogram was established to predict individual survival probability based on clinical characteristics and prognostic gene signature. Finally, the correlation between risk score and immune cell infiltration, genomic alteration, chemotherapy drug, and immune checkpoint blockade therapy was analyzed.

\section{Materials And Methods}




\subsection{Acquisition of colorectal cancer datasets and mitophagy-related genes}

RNA sequencing (RNA-seq), clinicopathological information, and somatic mutation data of 354 CRC patients were retrieved and downloaded from The Cancer Genome Atlas (TCGA, https://portal.gdc.cancer.gov/) program and cBioPortal for Cancer Genomics (https://cbioportal.org/). RNA-seq and clinicopathological information of 337 CRC patients (GSE17536 and GSE24551) were downloaded from the Gene Expression Omnibus (GEO, https://www.ncbi.nlm.nih.gov/geo/) database as the independent external validation cohorts. Forty-six mitophagy-related genes were obtained from the GO (http://geneontology.org/) and KEGG (https://www.genome.jp/kegg/) database. This study was carried out in accordance with relevant guidelines and regulations.

\subsection{Construction and validation of the prognostic gene signature}

Univariate Cox proportional hazard regression analysis was used to screen mitophagy-related genes

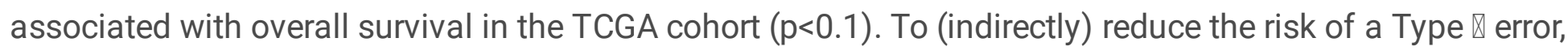
we set the significance level as 0.1 . The least absolute shrinkage and selection operator (LASSO) Cox regression model was performed to find the best gene signature from the genes obtained in univariate analysis by using the "glmnet" R package. The coefficient and expression of mitophagy related genes in the risk model were obtained, and the risk score of each patient was calculated. The formula is as follows: risk score $=\sum_{j=1}^{n} E x p r_{\text {genej }}{ }^{*} C_{0 e f_{\text {genej }}}$ with Expr indicating the level of gene expression and Coef representing the coefficient of gene. The median risk score was selected as the cutoff value to divide TCGA-CRC patients into high-risk group and low-risk group. Survival analysis between groups was based on overall survival, $p<0.05$ was considered statistically significant. Kaplan-Meier survival curves were plotted by the "survival" and "survminer" packages. To verify the accuracy and validity of signatures, "timeROC" R package was used to calculate the area under the curve (AUC) values at 1-, 3-, and 5-years derived from receiver operating characteristic (ROC) analysis. Prognostic gene signature was validated in two independent cohorts from GEO (GSE17536 and GSE24551). Univariate and multivariate Cox regression analyses were performed using clinical parameters and risk scores to assess the independent prognostic value of the signature.

\subsection{Enriched pathway analysis}

To analyze the potential functions of differential expressed genes of mitophagy-related signature, "clusterProfiler" R package was used for functional annotation of Gene Ontology (GO) and pathway enrichment analysis of Kyoto Encyclopedia of Genes and Genomes (KEGG). GO enrichment was carried out at three levels: cellular component (CC), biological process (BP) and molecular function (MF). KEGG analysis was performed from metabolic pathways and molecular mechanisms. To reveal biological process in the high-risk and low-risk groups, Gene Set Enrichment Analyses (GSEA) was performed by 
"ClusterProfiler" package in R studio. The false discovery rate (FDR) $q<0.01$ was considered statistically significant.

\subsection{Development of a nomogram}

Construction of a nomogram prognostic prediction model was based on risk scores, age, and $\mathrm{M}$ stage. AUC of the ROC curve, Harrell's concordance index (C-index), and calibration plots to compare predicted and observed overall survival were used to assess the performance of the prognostic nomogram. The Cindex ranges from 0.5 to 1.0 , with values of 0.5 and 1.0 representing a random chance and an excellent capacity for predicting survival with the model, respectively. The nomogram calibration curve was plotted to evaluate the concordance between actual and predicted survival.

\subsection{Evaluation of infiltrating immune cells}

CIBERSORT algorithm was used to calculate the tumor-infiltrating immune cells [17]. The algorithm is based on normalized gene expression data and an annotated gene signature matrix (LM22) that defines 22 immune cell subtypes. The LM22 file can be downloaded from The CIBERSORT web portal (https://cibersort.stanford.edu/). The perm was set to 1000. Samples with $p<0.05$ were considered qualified and screened for further analysis.

\subsection{Mutation analysis}

"maftools" R package was used to analyze the top 20 frequently mutated genes in the high-risk and lowrisk groups [18].

\subsection{Prediction of response of candidate small molecule drugs}

Using the Genomics of Drug Sensitivity in Cancer (GDSC, https://www.cancerrxgene.org/) database to estimate the sensitivity of each patient to chemotherapy drugs. The $\mathrm{IC}_{50}$ was quantified via "pRRophetic" $\mathrm{R}$ package. Screening method for candidate small molecule drugs referred to previously published article [19].

\subsection{Statistical analysis}

Data were processed, analyzed and presented using the R software (v.4.0.3) and related software packages. Kaplan-Meier curves were used to describe the relationship between patient survival time and survival probability. Visualization of risk-related information through charts, including risk score distribution, risk-related survival status, heat maps of prognostic genes, etc. The ROC analysis was used to analyze the sensitivity and specificity of survival prediction using the gene signature risk score. AUC served as an indicator of prognostic accuracy. Univariate and multivariate Cox regression analysis were used to verify the independence of signatures. And we used the "rms" R package to perform the nomogram. In addition, we assessed the correlation between the signature and clinical parameters. $\mathrm{P}<0.05$ was considered statistically significant. 


\section{Results}

\subsection{Construction of the prognostic signature based on mitophagy-related genes}

We searched and selected 46 genes related to mitophagy from the GO and KEGG database. In order to identify prognostic mitophagy related genes, we performed univariate Cox regression analysis on these genes, and a total of 44 genes were identified have correlation with the overall survival (OS) of TCGA-CRC (Figure 1A). Then, LASSO Cox regression analysis was applied, and 10 most prognostic genes (AMBRA1, ATG14, MAP1LC3A, MAP1LC3B, OPTN, VDAC1, ATG5, CSNK2A2, MFN1, TOMM22) associated with mitophagy were screened out (Figure 1B). compared with normal controls, the expression of AMBRA1, VDAC1, ATG5 and TOMM22 were significantly upregulated in tumors, while ATG14, OPTN, MAP1LC3A and MFN1 were significantly downregulated in tumors $(\mathrm{p}<0.0001$, Figure $1 \mathrm{C})$. The risk score was calculated according to their expression levels and Cox regression coefficients. The calculation formula is as follows, risk score $=$ expression level of $A M B R A 1 *(-0.0666731)+$ expression level of ATG14*0.26819842 + expression level of MAP1LC3B*0.03411786 + expression level of OPTN*0.26292307 + expression level of $\operatorname{VDAC1*(-0.16993759)}+$ expression level of $A T G 5^{\star}(-0.31642485)$ + expression level of CSNK2A2*0.07033702 + expression level of MAP1LC3A*0.09318544 + expression level of MFN1*(-0.27707284) + expression level of TOMM22*(-0.02760961). The characteristics of these genes were shown in Table 1.

Table 1

General characteristics of genes in the prognostic gene signature.

\begin{tabular}{|c|c|c|c|c|}
\hline $\begin{array}{l}\text { Gene } \\
\text { symbol }\end{array}$ & Full name & $\begin{array}{l}\text { Hazard } \\
\text { ratio }\end{array}$ & $\mathrm{p}$ value & $\begin{array}{l}\text { Risk } \\
\text { coefficient }\end{array}$ \\
\hline AMBRA1 & Autophagy and beclin 1 regulator 1 & 0.665151 & 0.071512 & -0.0666731 \\
\hline ATG14 & Autophagy related 14 & 1.753384 & 0.014580 & 0.26819842 \\
\hline MAP1LC3A & $\begin{array}{l}\text { Microtubule associated protein } 1 \text { light chain } \\
3 \text { alpha }\end{array}$ & 1.817103 & 0.017146 & 0.09318544 \\
\hline MAP1LC3B & $\begin{array}{l}\text { Microtubule associated protein } 1 \text { light chain } \\
3 \text { beta }\end{array}$ & 2.245661 & 0.000424 & 0.03411786 \\
\hline OPTN & Optineurin & 1.698439 & 0.043684 & 0.26292307 \\
\hline VDAC1 & Voltage dependent anion channel 1 & 0.531979 & 0.005017 & -0.16993759 \\
\hline ATG5 & Autophagy related 5 & 0.504291 & 0.002621 & -0.31642485 \\
\hline CSNK2A2 & Casein kinase 2 alpha 2 & 1.577459 & 0.069813 & 0.07033702 \\
\hline MFN1 & Mitofusin 1 & 0.685683 & 0.093788 & -0.27707284 \\
\hline TOMM22 & $\begin{array}{l}\text { Translocase of outer mitochondrial } \\
\text { membrane } 22\end{array}$ & 0.549266 & 0.007467 & -0.02760961 \\
\hline
\end{tabular}


The risk score for each patient was calculated according to the formula and applied to predict prognosis. Patients from the TCGA-CRC cohort were divided into high and low risk groups with the median risk score setting as the cutoff value. Kaplan-Meier curve based on log-rank test was used to identify prognostic differences between the high and low risk groups. Kaplan-Meier curve showed a significant difference in OS between the two groups of TCGA-CRC cohort, with patients in the high-risk group having a worse prognosis than that in the low-risk group (median OS 67.3 months vs not reached, $p=0.00059$ ) (Figure $2 A)$. Then time-dependent receiver operating characteristic (ROC) curve was used to verify the accuracy of the mitophagy gene signature. ROC curve showed that the area under the curves (AUC) of 1-, 3- and 5year survival time were $0.66,0.66$ and 0.64 (Figure 2B), respectively, indicating that the gene signature possessed a reliable ability for predicting the prognosis of $\mathrm{CRC}$ patients. We then analyzed the relationship between risk score distribution and survival status of CRC patients in the TCGA cohort (Figure 2C). As the patient's risk score increased, the patient's survival time decreased and the probability of death increased. The heatmap showed the expression profile of mitophagy related genes in high-risk and low-risk groups (Figure 2D). Genes with HR>1 was regarded as risk genes (ATG14, MAP1LC3B, OPTN, CSNK2A2, MAP1LC3A), and genes with $\mathrm{HR}<1$ were regarded as protective genes (AMBRA1, VDAC1, ATG5, MFN1, TOMM22). As shown in the figure, samples from in the high-risk group had a tendency to overexpress risk genes. In contrast, patients in the low-risk group were more likely to express protective genes.

\subsection{Validation of the prognostic gene signature in the independent CRC cohorts}

To validate the accuracy of this signature, we next evaluated the predictive ablity of the mitophagyrelated gene signature in different CRC cohorts (GSE17536 and GSE24551) from the GEO database. The two GEO-CRC patient cohorts were divided into high-risk group and low-risk group according to risk score respectively. Kaplan-Meier survival analysis showed significant differences in OS, and patients in the high-risk group both had a worse prognosis than those in the low-risk group (GSE17536, median OS 54.0 months vs not reached, $p=0.0082$; GSE24551, median OS 7.7 months vs not reached, $p=0.025$ ) (Figure 3A, 3D). ROC curves showed that the AUC of 1-, 3- and 5-year survival time of GSE17536 cohort were 0.54 , 0.55 and 0.55 , respectively (Figure 3B), and that of 1-, 2- and 3-year survival time of GSE24551 cohort were $0.65,0.57$ and 0.55 , respectively (Figure $3 \mathrm{E}$ ). The relationship between the distribution of risk score and survival status for the two GEO-CRC cohorts were shown in Figure $3 \mathrm{C}$ and $3 \mathrm{~F}$, which were similar to the results of TCGA-CRC cohort. In general, the mitophagy gene signature could stably and accurately predict the prognosis of CRC patients.

\subsection{Independent prognostic value of the prognostic gene signature}

Subsequently, we assessed the independent prognostic value of mitophagy gene signature. Univariate and multivariate Cox regression analyses were performed to evaluate whether signature-generated risk 
scores were independent of other clinical parameters (age, MSIsensor score, T, N, M stage) as prognostic factors for CRC patients. Univariate Cox regression analysis showed that in TCGA-CRC cohort, risk score, age, $\mathrm{T}, \mathrm{N}$, and $\mathrm{M}$ stage were significantly correlated with OS (Table 2). Multivariate Cox regression analysis showed that risk score was independently associated with OS in TCGA-CRC cohort $(H R=2.77$, $95 \% \mathrm{Cl}=1.27-6.04, \mathrm{p}=0.011$ ) (Table 2). These results further confirmed the high predictive accuracy of mitophagy gene signature, suggesting that the signature could be independently used to predict the prognosis of CRC patients.

Table 2

Univariate and multivariate Cox regression analysis of overall survival in TCGA.

\begin{tabular}{|lllll|}
\hline & UniCox & & MultiCox & \\
\hline & Hazard ratio & p value & Hazard ratio & p value \\
& $(95 \% \mathrm{Cl})$ & & $(95 \% \mathrm{Cl})$ & \\
\hline Age & $2.58(1.59-4.19)$ & $1.23 \mathrm{E}-04$ & $3.70(2.05-6.68)$ & $1.41 \mathrm{E}-05$ \\
\hline T stage & $2.21(1.02-4.82)$ & $4.52 \mathrm{E}-02$ & $1.59(0.55-4.56)$ & $3.90 \mathrm{E}-01$ \\
\hline N stage & $2.44(1.55-3.85)$ & $1.18 \mathrm{E}-04$ & $1.78(0.98-3.23)$ & $5.65 \mathrm{E}-02$ \\
\hline M stage & $3.38(1.98-5.78)$ & $8.75 \mathrm{E}-06$ & $3.54(1.87-6.70)$ & $1.00 \mathrm{E}-04$ \\
\hline MSlsensor score & $1.00(0.98-1.02)$ & $8.57 \mathrm{E}-01$ & $1.00(0.97-1.02)$ & $9.08 \mathrm{E}-01$ \\
\hline Risk score & $4.51(2.32-8.75)$ & $8.44 \mathrm{E}-06$ & $2.77(1.27-6.04)$ & $1.05 \mathrm{E}-02$ \\
\hline
\end{tabular}

\subsection{Relationships between the prognostic gene signature and clinical features}

The CRC cohort from TCGA include a total of 354 patients whose demographic and clinical features were listed in Supplementary Table 1. We further analyzed the relationship between the gene signature and clinical parameters of the TCGA-CRC patients. The results showed that the risk scores of patients with positive lymph node metastasis (N1\&2) were significantly higher than those without lymph node metastasis ( $p=0.0029$, Figure 4A). Similar results were also observed in T stage $(p=0.048$, Figure 4B). However, the risk score was not significantly associated with age and $M$ stage (Figure 4C, 4D). Based on these clinical features, patients were stratified to verify the effectiveness of the prognostic signature. The results showed that the signature could be well applied to each subgroup of $\mathrm{N}$ stage (Figure 4E, 4F), age (Figure 4G, 4H). But in $T$ and $M$ stage, the signature was more suitable for $T$ stage $3 \& 4$ and $M$ stage 0 (Figure 4I-L). In addition, the OS of patients in the high-risk group was worse than that of patients in the low-risk group in each stratum of these clinical parameters. In order to further explore the relationship between gene signature and microsatellite status in TCGA-CRC patients. We included 347 CRC patients with microsatellite instability (MSI) in the follow-up analysis, and the results showed that there was no significant correlation between risk score and MSI (Figure 4M). Meanwhile, patients from the high-risk group showed significantly worse prognosis than the low-risk group no matter whether were MSI-high or 
not (MSI-high, median OS 57.2 months vs not reached, $p=0.012$; MSI-low, median OS 61.8 months vs not reached, $p=0.0066$ ) (Figure $4 \mathrm{~N}, 40$ ). These results suggested that mitophagy related gene signature remained an important prognostic factor when stratifying CRC patients according to different clinical parameters.

\subsection{Enrichment analysis of the differentially expressed genes}

We performed enrichment analysis of differentially expressed genes (DEGs) in the high-risk and low-risk groups of the TCGA-CRC cohort. GO analysis simply annotated the gene functions, biological processes and localization in cells of the DEGs (Figure 5A). The biological process mainly involved ATP synthesis coupled electron transport, chromosome segregation, mitochondrial translational termination and cell cycle DNA replication. Gene products were mainly located in external encapsulating structure, collagen containing extracellular matrix and nuclear chromosome. Their functions mainly included extracellular matrix structural constituent and structural constituent of ribosome. KEGG analysis showed that these DEGs were mainly associated with ECM receptor interaction, focal adhesion and cell cycle pathways (Figure 5B). These genes were linked to each other and formed a tight protein-protein interaction network, as shown in Figure 5C. GSEA analysis was performed based on high-risk and low-risk groups. As shown in Figure 5D, the high-risk group was significantly correlated with extracellular matrix structural constituent (NES=3.2) and ECM receptor interaction (NES=2.48).

\subsection{Construction and validation of the prognostic prediction nomogram}

To better predict the prognosis of CRC patients with 1-, 3- and 5-year survival, we constructed a nomogram combining the risk score, age and $\mathrm{M}$ stage (Figure 6A). Calibration curves showed that for the TCGA-CRC cohort, actual and predicted survival matched very well (Figure 6B). The C-index of the nomogram was 0.77 ( $95 \% \mathrm{Cl}=0.71-0.83)$. The AUC of the 1-, 3- and 5-year overall survival predictions for the nomogram were $0.81,0.75$, and 0.68 , respectively (Figure $6 \mathrm{C}$ ).

\subsection{Relationship between mitophagy signature and immune cell infiltration}

To determine whether the gene signature could reflect the states of tumor immune microenvironment in CRC patients, we used CIBERSORT algorithm to calculate the proportion of 22 immune cells in each TCGA-CRC sample, and analyzed the relationship between immune cell infiltration and signature. Figure 7A showed the distribution of 22 immune cells in each sample. The results suggested that there were significant differences in the composition of immune cells in the two risk groups. Results showed that CD8+ T cells, regulatory $T$ cells and activated NK cells were significantly more abundant in the high-risk 
group, while the proportion of resting memory CD4+ T cells and resting mast cells were significantly higher in the low-risk group (Figure 7B).

\subsection{Mutation landscape of the prognostic gene signature}

By analyzing somatic mutation data from TCGA-CRC patients, we explored differences in genomic alterations between high-risk and low-risk groups. The oncoprint map showed the top 20 genes with the highest prevalence in high-risk and low-risk groups, respectively (Figure 8A, 8B). Missense mutations were the most common mutation type in both groups. Mutation frequency of $A P C, T P 53, T T N, K R A S, M U C 16$ and SYNE1 were all higher than $30 \%$ in the two groups. The mutations of PCLO, MUC5B and NEB genes were more prevalent in the high-risk group, while mutations of $D N A H 11, F B X W 7$ and $C S M D 3$ genes were more common in the low-risk group.

\subsection{Prediction of sensitivity to chemotherapy drugs and immune checkpoint blockade (ICB)}

We compared the differences in the half maximal inhibitory concentration $\left(\mathrm{IC}_{50}\right)$ levels of 10

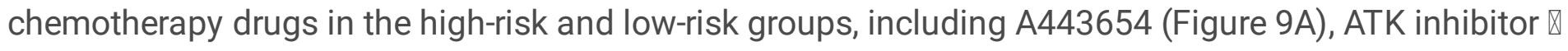
(Figure 9B), Motesanib (Figure 9C), ATRA (Figure 9D), Axitinib (Figure 9E), AZ628 (Figure 9F), Olaparib (Figure 9G), Selumetinib (Figure 9H), AZD6482 (Figure 9I) and AZD8055 (Figure 9J). Data showed that

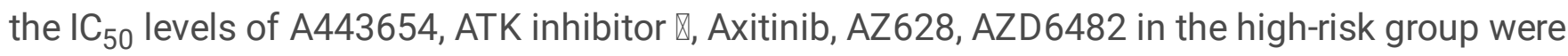
significantly higher than those in the low-risk group $(p<0.0001)$. On the contrary, there were significantly lower $\mathrm{IC}_{50}$ levels of Motesanib, ATRA, Olaparib, Selumetinib and AZD8055 in the high-risk group compared with the low-risk group $(p<0.0001)$, indicating that the high-risk group was more sensitive to these drugs. Tumor Immune Dysfunction and Exclusion (TIDE) algorithm (http://tide.dfci.harvard.edu/login/) was used to evaluate the responses of ICB therapies between the two groups. The TIDE score of high-risk patients was higher than that of low-risk patients (Figure 9K). In summary, patients in the high-risk group were more sensitive to Motesanib, ATRA, Olaparib, Selumetinib, AZD8055 chemotherapy and ICB therapy. In order to screen out potential small-molecule compounds that may be used to treat CRC patients, we identified a total of 14 potential small-molecule drugs, covering 15 mechanisms of action (Figure 9L), referring to the method used in previous report [19].

\section{Discussion}

$\mathrm{CRC}$ is one of the most common malignant tumors in the world, which has attracted much attention due to its high recurrence and metastasis rate, but low early detection rate and poor therapeutic choices [20, 21]. As increasing evidence revealing that mitophagy is closely associated with the progression and treatment of CRC [22-24], mitophagy related genes are a promising therapeutic target and prognostic indicator of CRC. However, understanding the role of mitophagy related gene signature available in CRC remains limited. 
In this study, we analyzed mitophagy related genes and identified 44 genes associated with CRC survival. Through LASSO regression analysis, 10 mitophagy related genes (AMBRA1, ATG14, MAP1LC3A, MAP1LC3B, OPTN, VDAC1, ATG5, CSNK2A2, MFN1, TOMM22) were screened out. Previous evidence has supported that some of these mitophagy-related genes involving in the development of CRC. For example, $A M B R A 1$ is a key regulator of autophagy and apoptosis in CRC cells, maintaining the balance between autophagy and apoptosis by interacting with Beclin1 [25]. Du et al. found that OPTN can be used to predict the prognosis of CRC patients [26]. VDAC1 mRNA and protein were significantly upregulated in CRC, and inhibition of VDAC1/AMPK/mTOR pathway could significantly inhibit the proliferation, metastasis and invasion of CRC cells [27]. ATG5 depletion can inhibit or promote CRC tumor growth [28]. A recent study suggested that $A T G 5$ acts as a tumor promoter in CRC metastasis and drug resistance [29]. CSNK2A2 suppresses apoptosis in CRC by desensitizing cells to TRAIL in a caspase-dependent manner but NF-K $\beta$ independent [30]. ATG14, MAP1LC3A and MAP1LC3B have also been confirmed to be abnormally expressed in CRC and affect the development of CRC tumors [31-33]. However, there is no previous study investigating the role of MFN1 and TOMM22 in the progression of CRC, we are the first study revealing the tumor suppressor role of these two genes, Especially TOMM22 which was overexpressed in the tumor samples. Enrichment analysis of DEGs also showed that these genes were enriched in "ECM structural constituent" and "ECM receptor interaction" pathways. Studies have shown that the abnormal expression of ECM protein is associated with the carcinogenesis and poor prognosis of CRC $[34,35]$.

The prognostic signature constructed based on the expression of the 10 genes above showed accurate and robust prognostic prediction capacity in TCGA-CRC and external independent GEO-CRC cohort. Patients with high-risk scores had significantly worse outcomes. When patients were stratified by traditional risk features such as age, TNM stage and MSI, mitophagy-related signature still retained its predictive ability to distinguish high-risk patients. Compared with the traditional risk features of age and $\mathrm{M}$ stage, the mitophagy-related signature was more accurate in predicting the prognosis of CRC patients (Supplementary Figure 1). In addition, we generated a nomogram to quantify the risk assessment and survival probability based on risk score, age and $\mathrm{M}$ stage. Compared to the above factors, the nomogram exhibited the highest accuracy and discrimination in survival prediction.

CRC patients will finally develop resistance to chemotherapy [36], thus novel therapeutic strategies are needed. Our analysis showed that high risk patients were more sensitive to Motesanib, ATRA, Olaparib, Selumetinib, and AZD8055. Among them, Motesanib has anti-proliferation, anti-angiogenesis and antiapoptosis effects on HT29 CRC cells when used alone, which may be a good choice for the treatment of CRC [37]. A growing number of vitro studies have shown that CRC cell lines are sensitive to PARP inhibitor Olaparib $[38,39]$. And a recent phase III LYNK-003 study demonstrated that Olaparib alone or in combination with bevacizumab as maintenance therapy after FOLFOX plus bevacizumab induction can prolong progression-free survival in advanced CRC patients compared with 5-FU plus bevacizumab maintenance [40]. In addition, combination Selumetinib and Cyclosporin A were well-tolerated in a previous Phase lb study and were active in $\mathrm{mCRC}$ [41]. Therefore, combination therapy with these agents in CRC patients may alleviate resistance mechanisms. 
Mismatch repair-deficient/microsatellite instable (dMMR/MSI) CRC tumors are highly infiltrated by immune cells [42], and the effectiveness of ICB in dMMR/MSI mCRC has been widely demonstrated [4346], but ICB has shown poor efficacy in pMMR/MSS CRC [47]. PMMR/MSS CRC patients present with distinct immune profiles, giving evidence of different immune escape mechanisms, which can be overcome by individualized immunotherapy [48]. The composition of the tumor microenvironment has been shown to influence ICB responses [49]. ICB works by reinvigorating an effective anti-tumor immune response by using immune cell infiltration (primarily T cells) within the tumor [49]. The degree of immune cell infiltration plays a crucial role in the prognosis of CRC patients [50,51]. A recent study showed that resting CD4 memory $T$ cells were the protective factor of CRC and could be used as an independent prognostic factor [52]. In various cancers, resting CD4 memory T cells were associated with increased overall survival, so the frequency of resting CD4 memory T cells predicted better survival [53-55]. And the level of mast cell infiltration in CRC is positively correlated with good prognosis [56]. In our study, infiltration levels of resting CD4 memory T cells and resting mast cells were significantly lower in the high-risk group with poor prognosis than in the low-risk group. Different numbers, phenotypes, and localization of tumor-infiltrating lymphocytes are not only key regulators of disease progression, but also potential biomarkers for predicting immunotherapy response [57]. This shows the potential of our signature in predicting tumor immune microenvironment of CRC, which might benefit the immunotherapy of this malignancy. TIDE computational method, which integrates the expression signatures of $\mathrm{T}$ cell dysfunction and $T$ cell exclusion to simulate tumor immune escape, can predict clinical response to ICB based on pre-treatment tumor profiles [58]. We then used the TIDE method to predict the response of highrisk score and low-risk score CRC patients to ICB therapy. TIDE score was significantly higher in high-risk group, suggesting that high-risk score patients were more sensitive to ICB therapy. Thus, our signature had the potency for assisting oncologists to decide which patients are likely to respond to ICB in order to take the best course of treatment.

In conclusion, we constructed a signature that could predict the prognosis of CRC patients based on mitophagy related genes and could be used as an independent prognostic factor. The signature could also reflect the immune status of CRC patients to a certain extent. Our findings suggest that the prognostic signature may be useful for personalized treatment in clinical settings.

\section{Declarations}

\section{Consent for publication}

Not applicable.

\section{Data availability statement}

The data that support the findings of this study are available from the corresponding author upon reasonable request.

\section{Declaration of competing interest}


The authors confirm that there are no conflicts of interest.

\section{Funding}

None.

\section{Author contributions}

Cong Zhang and Cailing Zeng conceived, designed and wrote the manuscript of this study. Shaoquan Xiong and Zewei Zhao obtained the datasets and conducted data analysis. Guoyu Wu revised the manuscript. All authors reviewed and approved the final manuscript.

\section{Acknowledgements}

We are grateful of all participants in this study.

\section{References}

1. Bray F, Ferlay J, Soerjomataram I, Siegel RL, Torre LA, Jemal A: Global cancer statistics 2018: GLOBOCAN estimates of incidence and mortality worldwide for 36 cancers in 185 countries. CA: a cancer journal for clinicians 2018, 68(6):394-424.

2. Inamura K: Colorectal Cancers: An Update on Their Molecular Pathology. Cancers 2018, 10(1).

3. Thanikachalam K, Khan G: Colorectal Cancer and Nutrition. Nutrients 2019, 11(1).

4. De Greef K, Rolfo C, Russo A, Chapelle T, Bronte G, Passiglia F, Coelho A, Papadimitriou K, Peeters M: Multisciplinary management of patients with liver metastasis from colorectal cancer. World journal of gastroenterology 2016, 22(32):7215-7225.

5. Edwards BK, Ward E, Kohler BA, Eheman C, Zauber AG, Anderson RN, Jemal A, Schymura MJ, Lansdorp-Vogelaar I, Seeff LC et al: Annual report to the nation on the status of cancer, 1975-2006, featuring colorectal cancer trends and impact of interventions (risk factors, screening, and treatment) to reduce future rates. Cancer 2010, 116(3):544-573.

6. Siegel R, Desantis C, Jemal A: Colorectal cancer statistics, 2014. CA: a cancer journal for clinicians 2014, 64(2):104-117.

7. van der Stok EP, Spaander MCW, Grünhagen DJ, Verhoef C, Kuipers EJ: Surveillance after curative treatment for colorectal cancer. Nature reviews Clinical oncology 2017, 14(5):297-315.

8. Markman JL, Shiao SL: Impact of the immune system and immunotherapy in colorectal cancer. Journal of gastrointestinal oncology 2015, 6(2):208-223.

9. Singh PP, Sharma PK, Krishnan G, Lockhart AC: Immune checkpoints and immunotherapy for colorectal cancer. Gastroenterology report 2015, 3(4):289-297.

10. Jiao Q, Ren Y, Ariston Gabrie AN, Wang Q, Wang Y, Du L, Liu X, Wang C, Wang YS: Advances of immune checkpoints in colorectal cancer treatment. Biomedicine \& pharmacotherapy = Biomedecine \& pharmacotherapie 2020, 123:109745. 
11. Ganesh K, Stadler ZK, Cercek A, Mendelsohn RB, Shia J, Segal NH, Diaz LA, Jr.: Immunotherapy in colorectal cancer: rationale, challenges and potential. Nature reviews Gastroenterology \& hepatology 2019, 16(6):361-375.

12. Onishi M, Yamano K, Sato M, Matsuda N, Okamoto K: Molecular mechanisms and physiological functions of mitophagy. The EMBO journal 2021, 40(3):e104705.

13. Doblado L, Lueck C, Rey C, Samhan-Arias AK, Prieto I, Stacchiotti A, Monsalve M: Mitophagy in Human Diseases. International journal of molecular sciences 2021, 22(8).

14. Sowter HM, Ratcliffe PJ, Watson P, Greenberg AH, Harris AL: HIF-1-dependent regulation of hypoxic induction of the cell death factors BNIP3 and NIX in human tumors. Cancer research 2001, 61(18):6669-6673.

15. Chen WT, Yang HB, Ke TW, Liao WL, Hung SY: Serum DJ-1 Is a Biomarker of Colorectal Cancer and DJ-1 Activates Mitophagy to Promote Colorectal Cancer Progression. Cancers 2021, 13(16).

16. He L, Gu K: Tanshinone IIA regulates colorectal cancer apoptosis via attenuation of Parkin-mediated mitophagy by suppressing AMPK/Skp2 pathways. Molecular medicine reports 2018, 18(2):16921703.

17. Newman AM, Liu CL, Green MR, Gentles AJ, Feng W, Xu Y, Hoang CD, Diehn M, Alizadeh AA: Robust enumeration of cell subsets from tissue expression profiles. Nature methods 2015, 12(5):453-457.

18. Skidmore ZL, Wagner AH, Lesurf R, Campbell KM, Kunisaki J, Griffith OL, Griffith M: GenVisR: Genomic Visualizations in R. Bioinformatics (Oxford, England) 2016, 32(19):3012-3014.

19. Bai D, Feng H, Yang J, Yin A, Lin X, Qian A, Sugiyama H: Genomic analysis uncovers prognostic and immunogenic characteristics of ferroptosis for clear cell renal cell carcinoma. Molecular therapy Nucleic acids 2021, 25:186-197.

20. Siegel RL, Miller KD, Fedewa SA, Ahnen DJ, Meester RGS, Barzi A, Jemal A: Colorectal cancer statistics, 2017. CA: a cancer journal for clinicians 2017, 67(3):177-193.

21. Long J, He Q, Yin Y, Lei X, Li Z, Zhu W: The effect of miRNA and autophagy on colorectal cancer. Cell proliferation 2020, 53(10):e12900.

22. Satoh K, Yachida S, Sugimoto M, Oshima M, Nakagawa T, Akamoto S, Tabata S, Saitoh K, Kato K, Sato $S$ et al: Global metabolic reprogramming of colorectal cancer occurs at adenoma stage and is induced by MYC. Proceedings of the National Academy of Sciences of the United States of America 2017, 114(37):E7697-e7706.

23. Ziegler PK, Bollrath J, Pallangyo CK, Matsutani T, Canli Ö, De Oliveira T, Diamanti MA, Müller N, Gamrekelashvili J, Putoczki T et al: Mitophagy in Intestinal Epithelial Cells Triggers Adaptive Immunity during Tumorigenesis. Cell 2018, 174(1):88-101.e116.

24. Yin K, Lee J, Liu Z, Kim H, Martin DR, Wu D, Liu M, Xue X: Mitophagy protein PINK1 suppresses colon tumor growth by metabolic reprogramming via p53 activation and reducing acetyl-CoA production. Cell death and differentiation 2021, 28(8):2421-2435.

25. Gu W, Wan D, Qian Q, Yi B, He Z, Gu Y, Wang L, He S: Ambra1 is an essential regulator of autophagy and apoptosis in SW620 cells: pro-survival role of Ambra1. PloS one 2014, 9(2):e90151. 
26. Du W, Hua F, Li X, Zhang J, Li S, Wang W, Zhou J, Wang W, Liao P, Yan Y et al: Loss of Optineurin Drives Cancer Immune Evasion via Palmitoylation-Dependent IFNGR1 Lysosomal Sorting and Degradation. Cancer discovery 2021, 11(7):1826-1843.

27. Liu X, He B, Xu T, Pan Y, Hu X, Chen X, Wang S: MiR-490-3p Functions As a Tumor Suppressor by Inhibiting Oncogene VDAC1 Expression in Colorectal Cancer. Journal of Cancer 2018, 9(7):12181230.

28. Kuipers EJ, Grady WM, Lieberman D, Seufferlein T, Sung JJ, Boelens PG, van de Velde CJ, Watanabe T: Colorectal cancer. Nature reviews Disease primers 2015, 1:15065.

29. Hu WH, Yang WC, Liu PF, Liu TT, Morgan P, Tsai WL, Pan HW, Lee CH, Shu CW: Clinicopathological Association of Autophagy Related 5 Protein with Prognosis of Colorectal Cancer. Diagnostics (Basel, Switzerland) 2021, 11(5).

30. Izeradjene K, Douglas L, Delaney A, Houghton JA: Casein kinase II (CK2) enhances death-inducing signaling complex (DISC) activity in TRAIL-induced apoptosis in human colon carcinoma cell lines. Oncogene 2005, 24(12):2050-2058.

31. Awi NJ, Armon S, Peh KB, Peh SC, Teow SY: High expression of LC3A, LC3B, and p62/SQSTM1 autophagic proteins in human colonic ganglion cells. The Malaysian journal of pathology 2020, 42(1):85-90.

32. Guo GF, Wang YX, Zhang YJ, Chen XX, Lu JB, Wang HH, Jiang C, Qiu HQ, Xia LP: Predictive and prognostic implications of 4E-BP1, Beclin-1, and LC3 for cetuximab treatment combined with chemotherapy in advanced colorectal cancer with wild-type KRAS: Analysis from real-world data. World journal of gastroenterology 2019, 25(15):1840-1853.

33. Han Y, Zhou S, Wang X, Mao E, Huang L: SNHG14 stimulates cell autophagy to facilitate cisplatin resistance of colorectal cancer by regulating miR-186/ATG14 axis. Biomedicine \& pharmacotherapy = Biomedecine \& pharmacotherapie 2020, 121:109580.

34. Wu Y, Xu Y: Integrated bioinformatics analysis of expression and gene regulation network of COL12A1 in colorectal cancer. Cancer medicine 2020, 9(13):4743-4755.

35. Lascorz J, Bevier M, W VS, Kalthoff H, Aselmann H, Beckmann J, Egberts J, Buch S, Becker T, Schreiber $S$ et al: Association study identifying polymorphisms in CD47 and other extracellular matrix pathway genes as putative prognostic markers for colorectal cancer. International journal of colorectal disease 2013, 28(2):173-181.

36. Picco G, Cattaneo CM, van Vliet EJ, Crisafulli G, Rospo G, Consonni S, Vieira SF, Rodríguez IS, Cancelliere C, Banerjee R et al: Werner Helicase Is a Synthetic-Lethal Vulnerability in Mismatch Repair-Deficient Colorectal Cancer Refractory to Targeted Therapies, Chemotherapy, and Immunotherapy. Cancer discovery 2021, 11(8):1923-1937.

37. Kaya TT, Altun A, Turgut NH, Ataseven H, Koyluoglu G: Effects of a Multikinase Inhibitor Motesanib (AMG 706) Alone and Combined with the Selective DuP-697 COX-2 Inhibitor on Colorectal Cancer Cells. Asian Pacific journal of cancer prevention: APJCP 2016, 17(3):1103-1110. 
38. Wang C, Jette N, Moussienko D, Bebb DG, Lees-Miller SP: ATM-Deficient Colorectal Cancer Cells Are Sensitive to the PARP Inhibitor Olaparib. Translational oncology 2017, 10(2):190-196.

39. Arena S, Corti G, Durinikova E, Montone M, Reilly NM, Russo M, Lorenzato A, Arcella P, Lazzari L, Rospo $\mathrm{G}$ et al: A Subset of Colorectal Cancers with Cross-Sensitivity to Olaparib and Oxaliplatin. Clinical cancer research: an official journal of the American Association for Cancer Research 2020, 26(6):1372-1384.

40. Kim TW, Taieb J, Gurary EB, Lerman N, Cui K, Yoshino T: Olaparib with or without bevacizumab or bevacizumab and 5-fluorouracil in advanced colorectal cancer. Phase III LYNK-003. Future oncology (London, England) 2021.

41. Krishnamurthy A, Dasari A, Noonan AM, Mehnert JM, Lockhart AC, Leong S, Capasso A, Stein MN, Sanoff HK, Lee JJ et al: Phase Ib Results of the Rational Combination of Selumetinib and Cyclosporin A in Advanced Solid Tumors with an Expansion Cohort in Metastatic Colorectal Cancer. Cancer research 2018, 78(18):5398-5407.

42. Jass JR: Classification of colorectal cancer based on correlation of clinical, morphological and molecular features. Histopathology 2007, 50(1):113-130.

43. Le DT, Uram JN, Wang H, Bartlett BR, Kemberling H, Eyring AD, Skora AD, Luber BS, Azad NS, Laheru $\mathrm{D}$ et al: PD-1 Blockade in Tumors with Mismatch-Repair Deficiency. The New England journal of medicine 2015, 372(26):2509-2520.

44. Overman MJ, Lonardi S, Wong KYM, Lenz HJ, Gelsomino F, Aglietta M, Morse MA, Van Cutsem E, McDermott R, Hill A et al: Durable Clinical Benefit With Nivolumab Plus Ipilimumab in DNA Mismatch Repair-Deficient/Microsatellite Instability-High Metastatic Colorectal Cancer. Journal of clinical oncology: official journal of the American Society of Clinical Oncology 2018, 36(8):773-779.

45. Overman MJ, McDermott R, Leach JL, Lonardi S, Lenz HJ, Morse MA, Desai J, Hill A, Axelson M, Moss RA et al: Nivolumab in patients with metastatic DNA mismatch repair-deficient or microsatellite instability-high colorectal cancer (CheckMate 142): an open-label, multicentre, phase 2 study. The Lancet Oncology 2017, 18(9):1182-1191.

46. Le DT, Durham JN, Smith KN, Wang H, Bartlett BR, Aulakh LK, Lu S, Kemberling H, Wilt C, Luber BS et al: Mismatch repair deficiency predicts response of solid tumors to PD-1 blockade. Science (New York, NY) 2017, 357(6349):409-413.

47. Eng C, Kim TW, Bendell J, Argilés G, Tebbutt NC, Di Bartolomeo M, Falcone A, Fakih M, Kozloff M, Segal $\mathrm{NH}$ et al: Atezolizumab with or without cobimetinib versus regorafenib in previously treated metastatic colorectal cancer (IMblaze370): a multicentre, open-label, phase 3, randomised, controlled trial. The Lancet Oncology 2019, 20(6):849-861.

48. Cohen R, Rousseau B, Vidal J, Colle R, Diaz LA, Jr., André T: Immune Checkpoint Inhibition in Colorectal Cancer: Microsatellite Instability and Beyond. Targeted oncology 2020, 15(1):11-24.

49. Petitprez F, Meylan M, de Reyniès A, Sautès-Fridman C, Fridman WH: The Tumor Microenvironment in the Response to Immune Checkpoint Blockade Therapies. Frontiers in immunology 2020, 11:784. 
50. Peng D, Wang L, Li H, Cai C, Tan Y, Xu B, Le H: An immune infiltration signature to predict the overall survival of patients with colon cancer. IUBMB life 2019, 71(11):1760-1770.

51. Ge P, Wang W, Li L, Zhang G, Gao Z, Tang Z, Dang X, Wu Y: Profiles of immune cell infiltration and immune-related genes in the tumor microenvironment of colorectal cancer. Biomedicine \& pharmacotherapy $=$ Biomedecine \& pharmacotherapie 2019, 118:109228.

52. Zhang X, Quan F, Xu J, Xiao Y, Li X, Li Y: Combination of multiple tumor-infiltrating immune cells predicts clinical outcome in colon cancer. Clinical immunology (Orlando, Fla) 2020, 215:108412.

53. Kim HM, Koo JS: Clinicopathologic Characteristics of Breast Cancer According to the Infiltrating Immune Cell Subtypes. International journal of molecular sciences 2020, 21(12).

54. Liu J, Chen X, Jiang Y, Cheng W: Development of an immune gene prognostic classifier for survival prediction and respond to immunocheckpoint inhibitor therapy/chemotherapy in endometrial cancer. International immunopharmacology 2020, 86:106735.

55. Tamminga M, Hiltermann TJN, Schuuring E, Timens W, Fehrmann RS, Groen HJ: Immune microenvironment composition in non-small cell lung cancer and its association with survival. Clinical \& translational immunology 2020, 9(6):e1142.

56. Nielsen HJ, Hansen U, Christensen IJ, Reimert CM, Brünner N, Moesgaard F: Independent prognostic value of eosinophil and mast cell infiltration in colorectal cancer tissue. The Journal of pathology 1999, 189(4):487-495.

57. Maibach F, Sadozai H, Seyed Jafari SM, Hunger RE, Schenk M: Tumor-Infiltrating Lymphocytes and Their Prognostic Value in Cutaneous Melanoma. Frontiers in immunology 2020, 11:2105.

58. Jiang P, Gu S, Pan D, Fu J, Sahu A, Hu X, Li Z, Traugh N, Bu X, Li B et al: Signatures of T cell dysfunction and exclusion predict cancer immunotherapy response. Nature medicine 2018, 24(10):1550-1558.

\section{Figures}



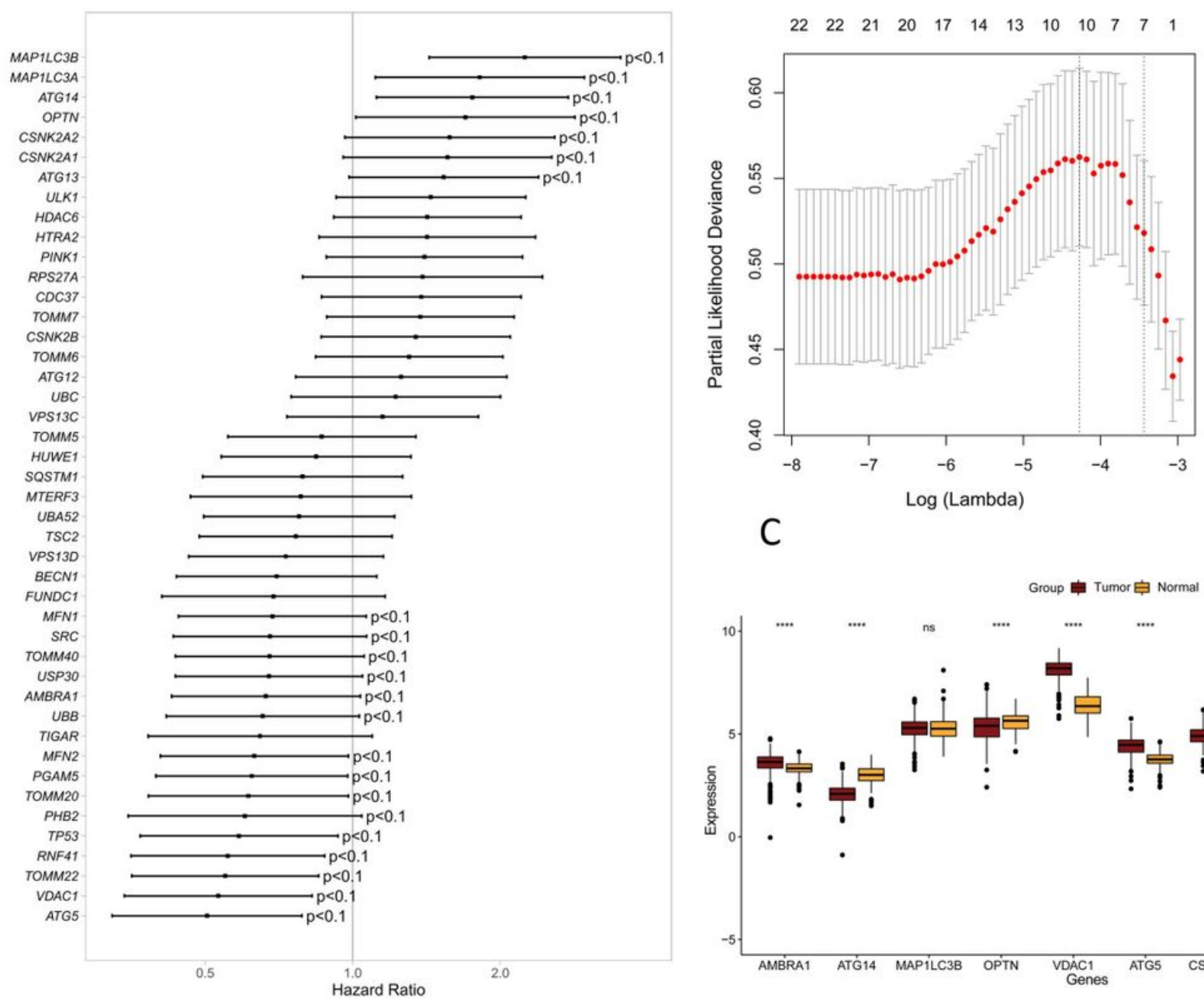

C

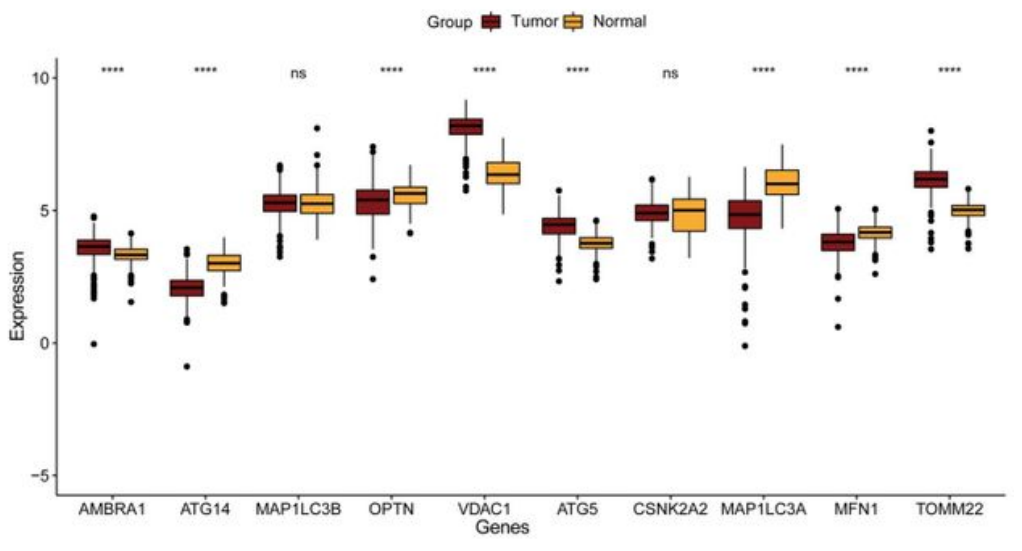

Figure 1

Establishment of the prognostic gene signature based on 10-mitophagy-related genes. (A) The mitophagy related genes associated with CRC survival were selected by univariate Cox regression analysis. (B) Cross-validation for tuning parameter (lambda) screening in the least absolute shrinkage and selection operator (LASSO) regression model based on minimum criteria for OS. (C) Differential expression of the 10 selected genes between normal and CRC tissues. 
A

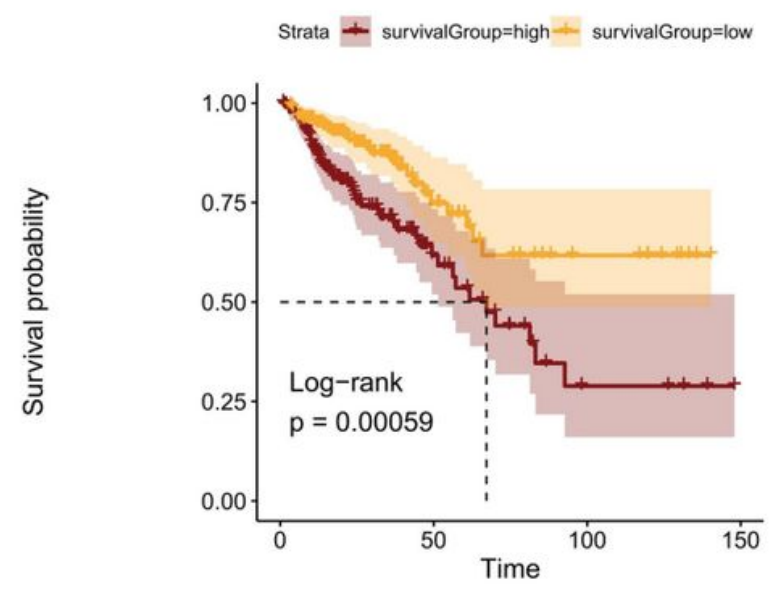

Number at risk

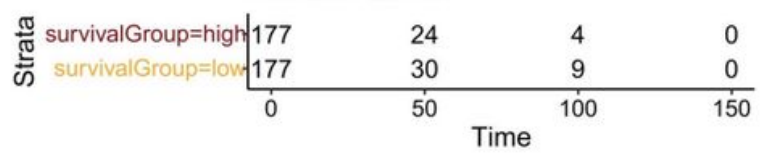

B

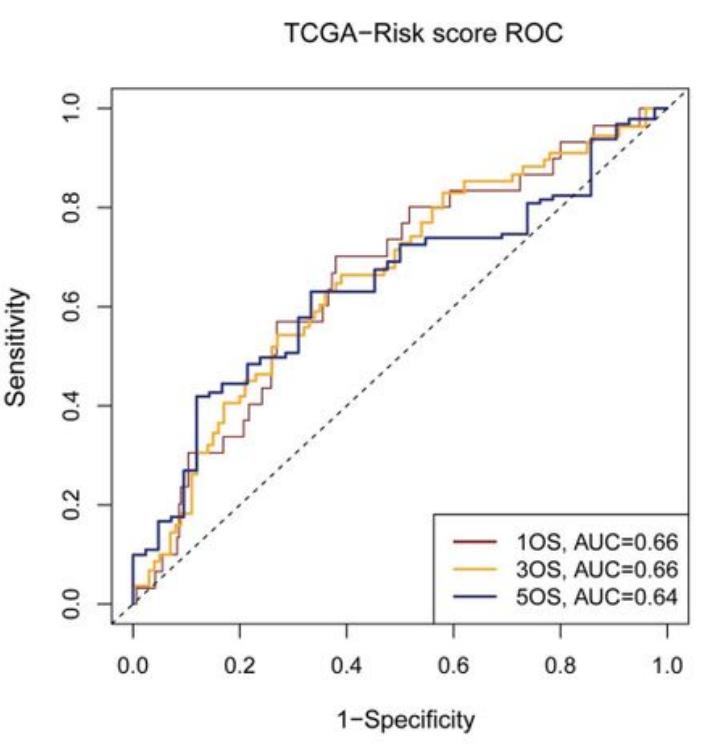

C
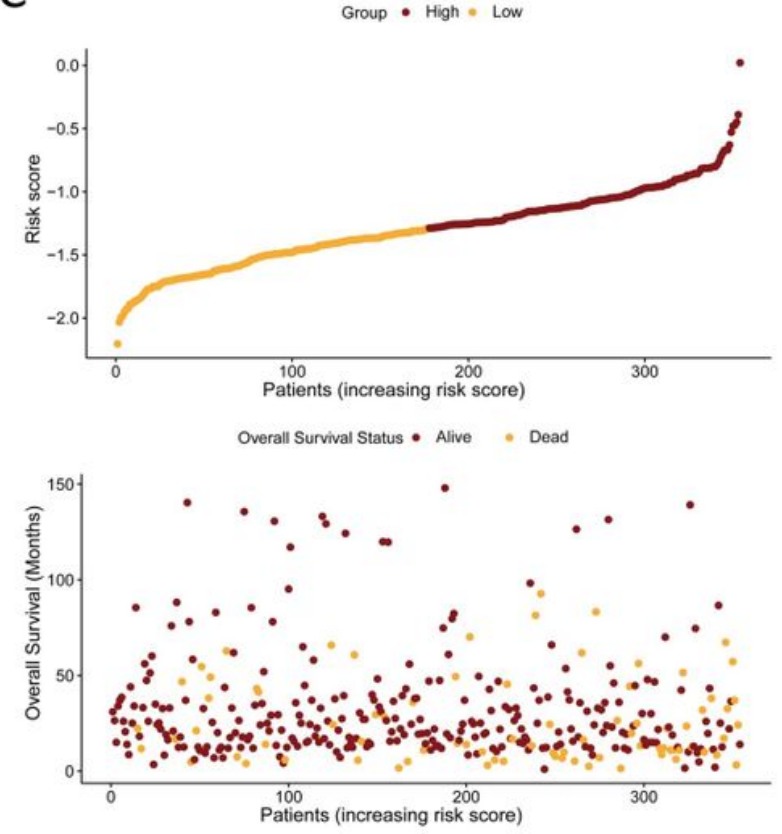

D

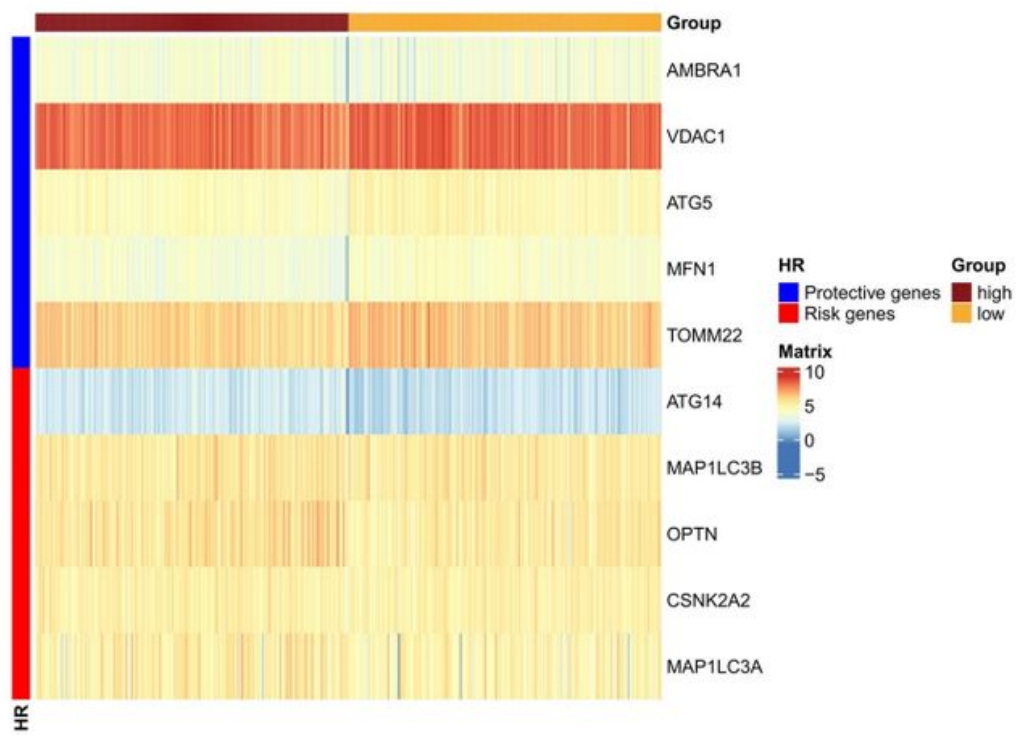

Figure 2

The prognostic value of the signature in the TCGA cohort. (A) Kaplan-Meier survival analysis for TCGACRC patients with high and low risk scores. (B) Time dependent ROC curve analysis of the risk score in TCGA cohort. (C) Risk score distribution and survival status for TCGA-CRC patients in high-risk and lowrisk groups. (D) Heatmap of the mitophagy related genes expression profile for TCGA-CRC patients in high-risk and low-risk groups. 
A
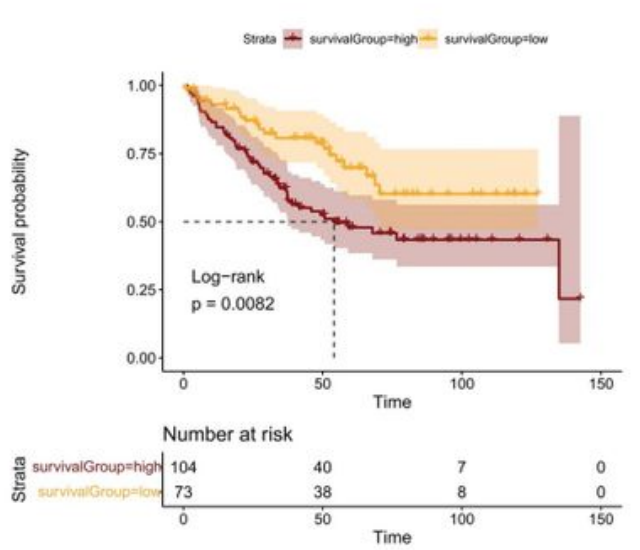

B

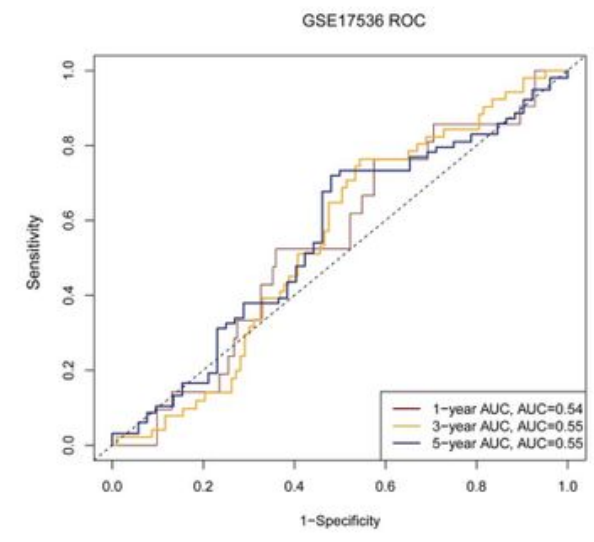

C

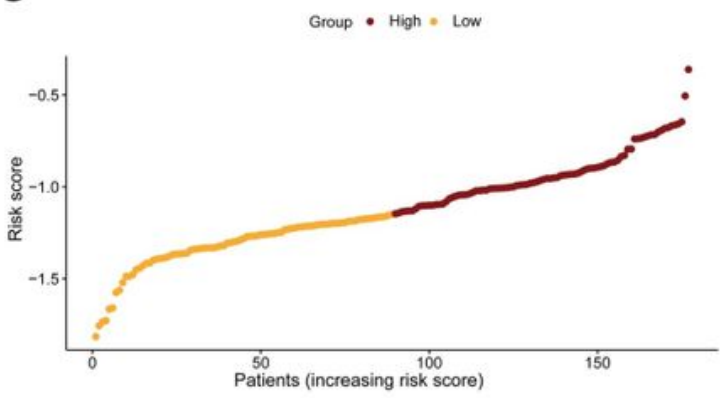

status - Alive - Dead

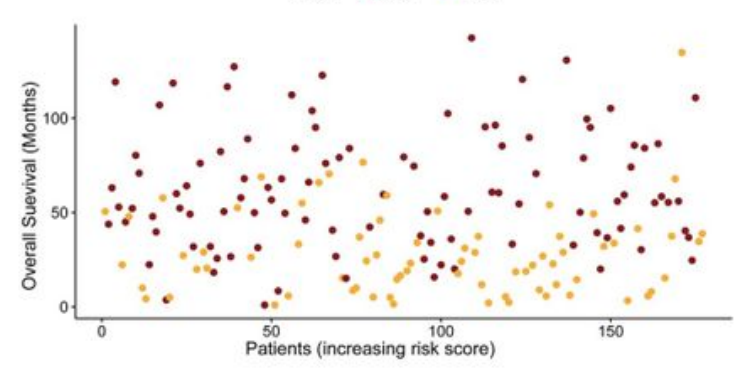

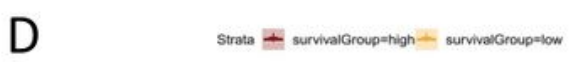
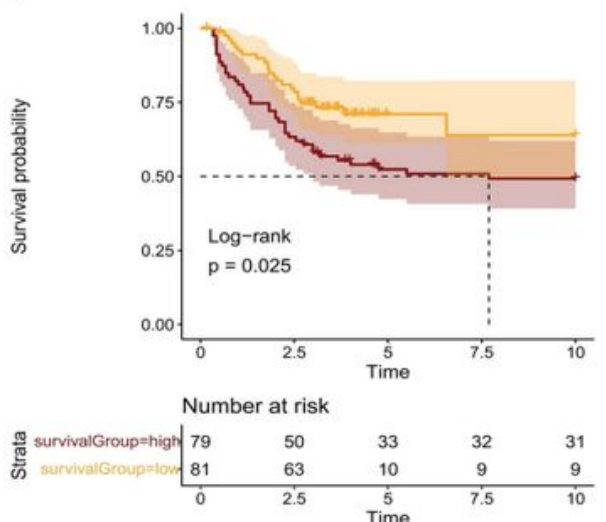

E

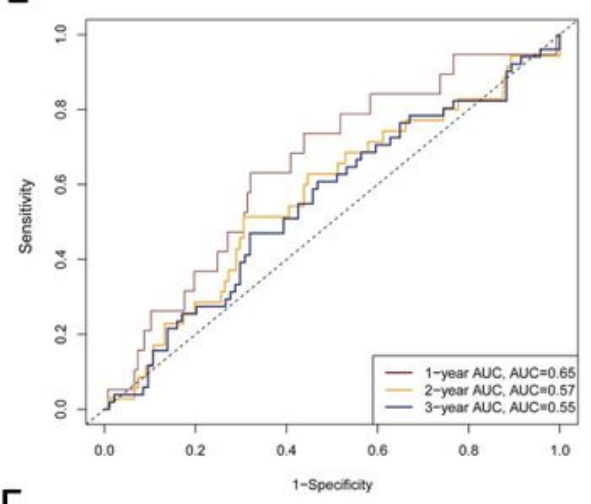

$\mathrm{F}$

Group - High - Low
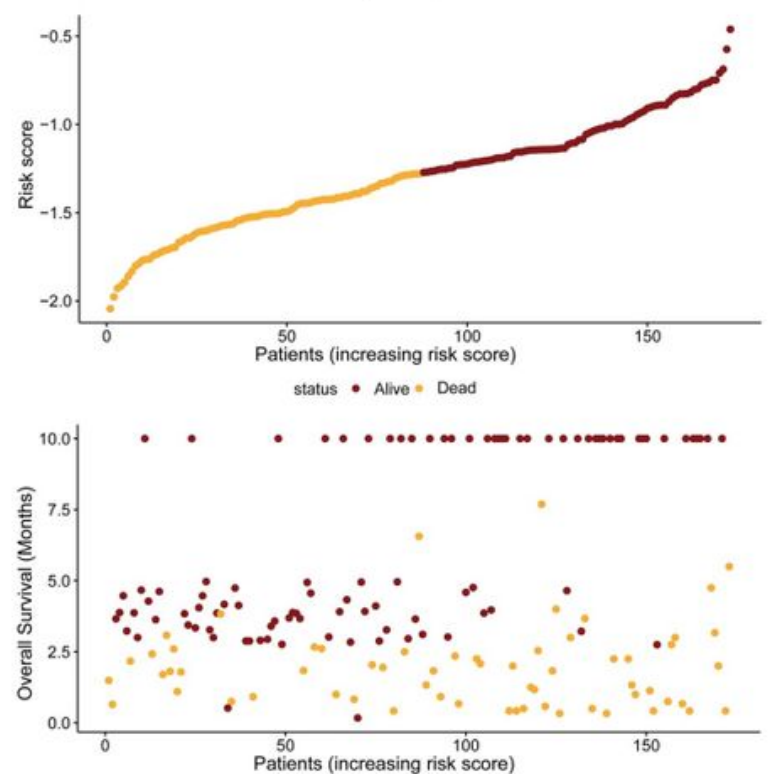

\section{Figure 3}

Validation of the prognostic signature in GSE17536 and GSE24551 cohort. (A, D) Kaplan-Meier survival analysis for GEO-CRC patients with high and low risk scores. $(B, E)$ Time dependent ROC curve analysis of the risk score in GEO cohort. (C, F) Risk score distribution and survival status for GEO-CRC patients in high-risk and low-risk groups. 

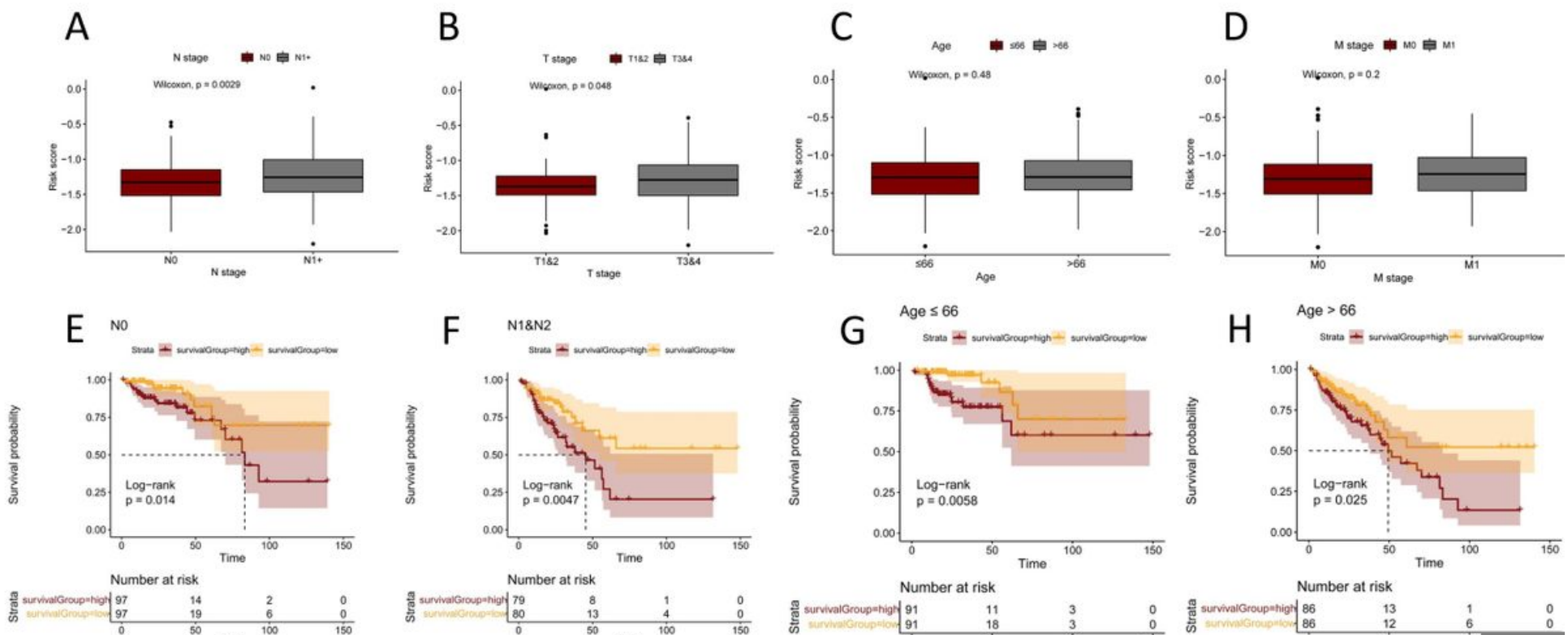

\section{Figure 4}

Association between pathologic characteristics and the prognostic signature in the TCGA cohort. (A-D, M) Distribution of the risk score in TCGA-CRC patients stratified by $\mathrm{N}$ stage, $\mathrm{T}$ stage, age, $\mathrm{M}$ stage and MSI. $(E-L, N, 0)$ The high-risk group showed a poor prognosis than the low-risk group in different clinical stratification. 
A

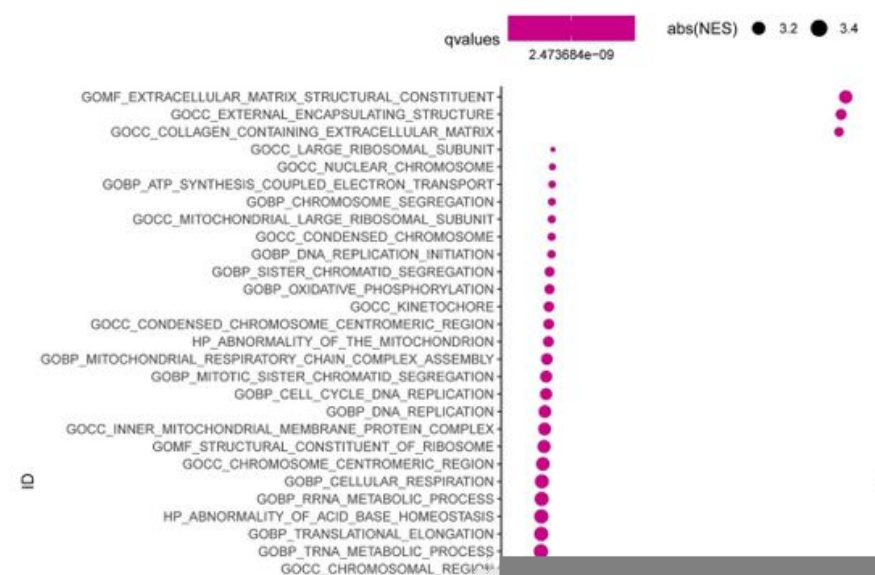

B

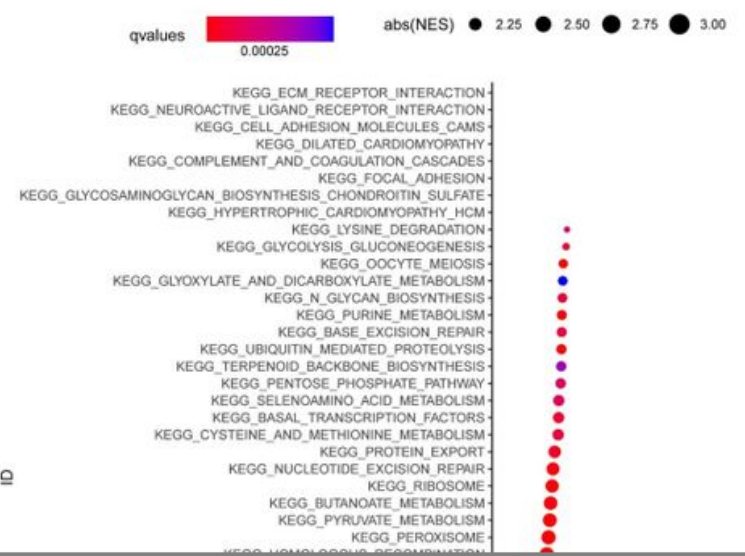

Figure 5

GO, KEGG, protein-protein interaction (PPI) network and GSEA analysis. (A) GO and (B) KEGG analysis of 10 mitophagy related genes. (C) PPI network of mitophagy related genes, revealing their intrinsic connections. (D) GSEA analysis of the mitophagy related genes between high and low risk groups. 

A
Points

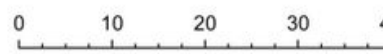
40,50
$50 \quad 60$
Risk score

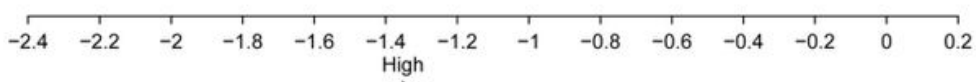
Age
M stage
Low

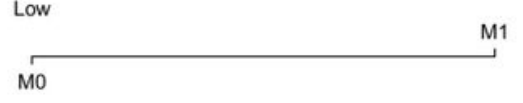
Total Points
Linear Predictor

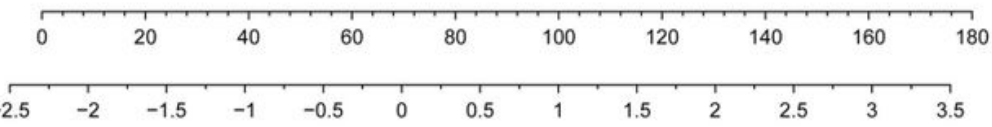
1-year survival
3-year survival
5-year survival

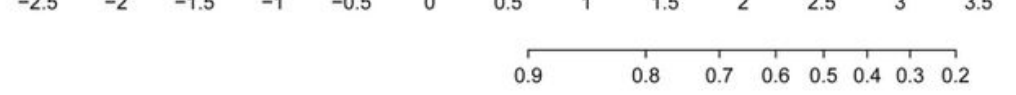

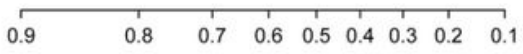

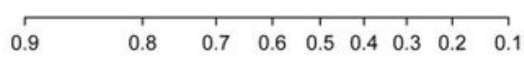

B

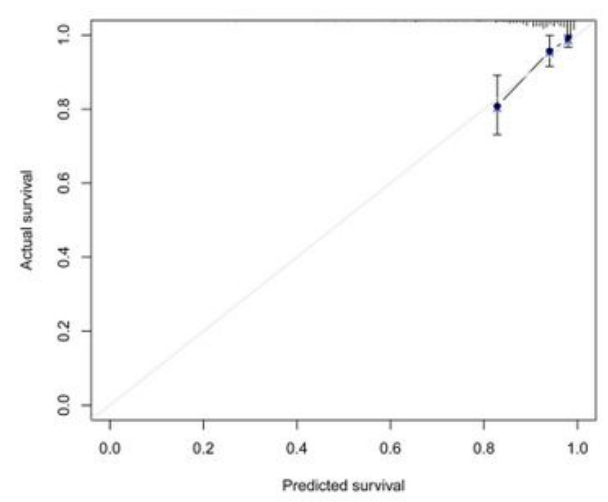

3.year calibration curve

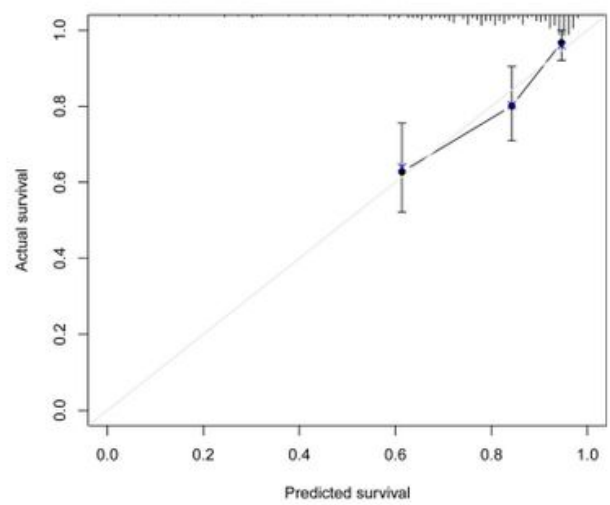

Nomogram ROC

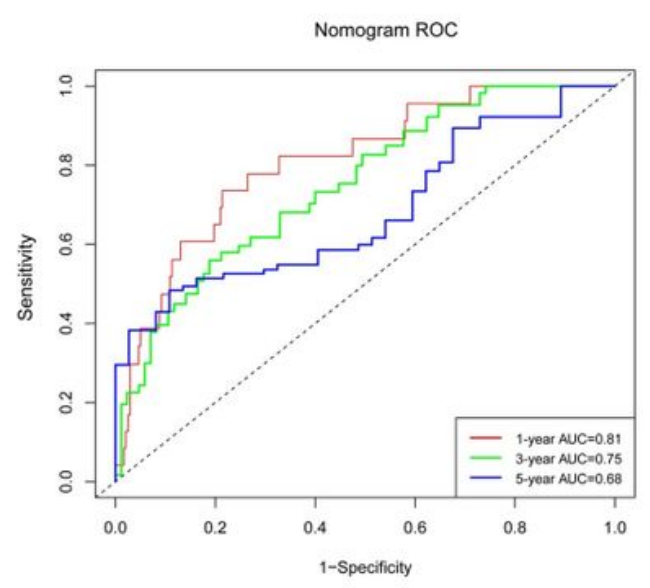

5-year calibration curve

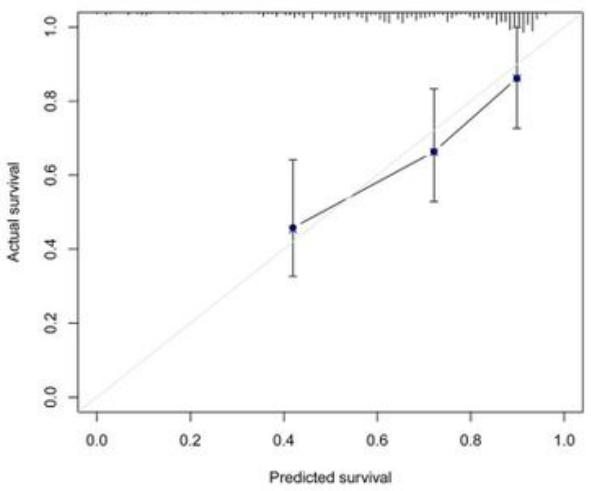

C

Figure 6

The nomogram for predicting overall survival of CRC patients. (A) The nomogram integrating the signature risk score with the pathologic characteristics for predicting OS. (B) The calibration curve for the nomogram in TCGA cohort. (C) Time dependent ROC curve analysis of the nomogram in TCGA cohort. 
A

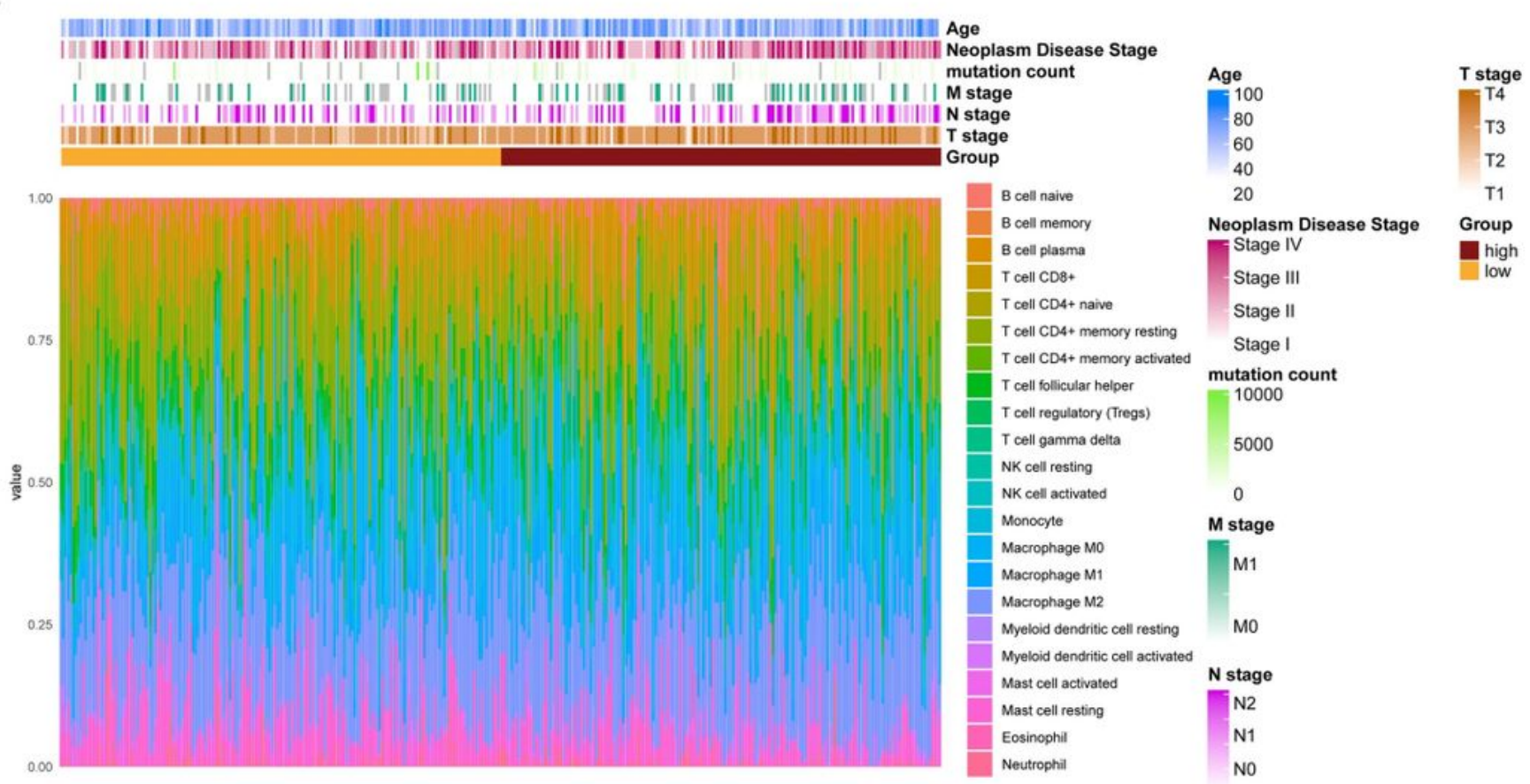

B

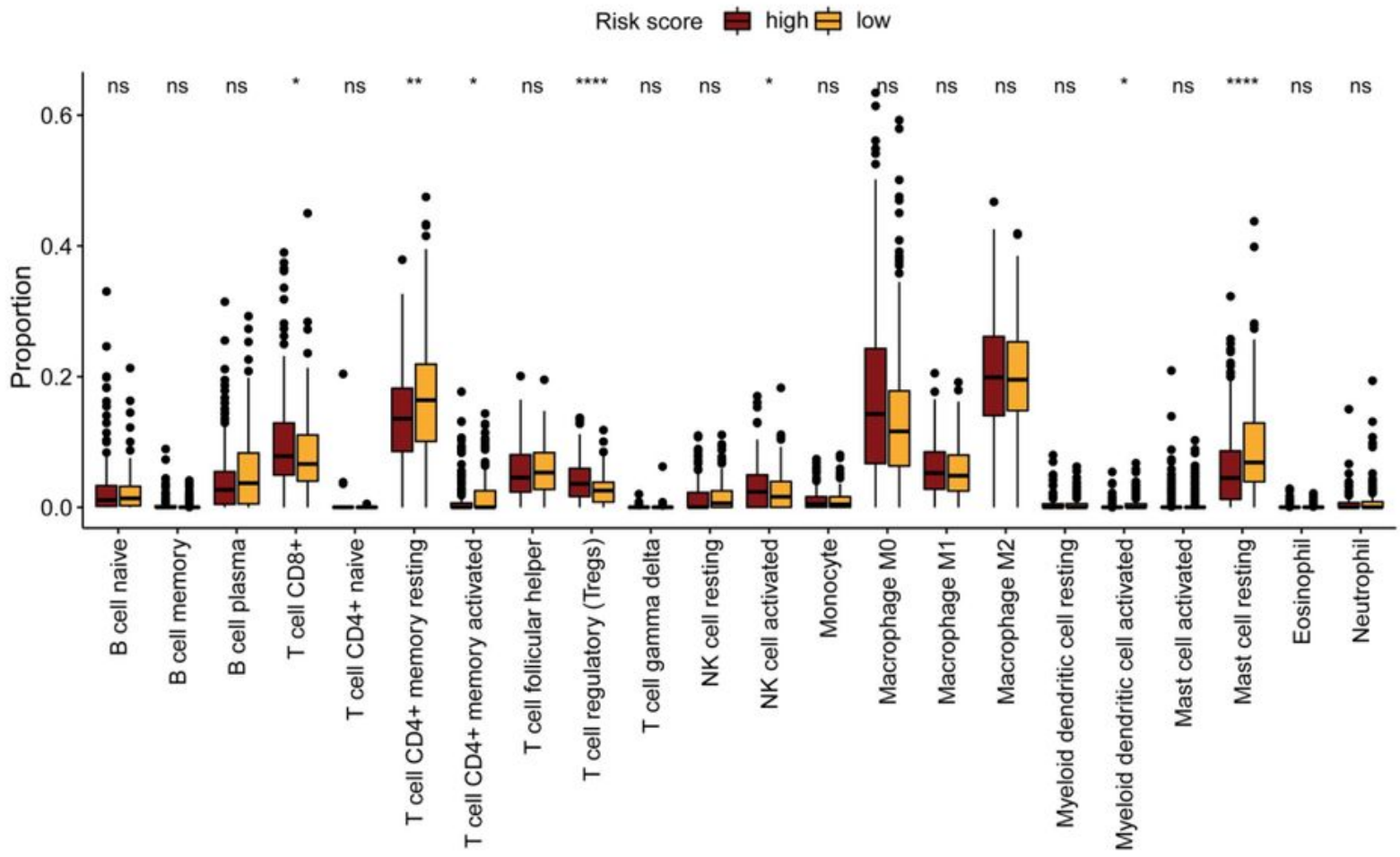

Figure 7

The association of the prognostic signature and immune cell infiltration. (A) The distribution of 22 immune cells in each TCGA-CRC patient. (B) Boxplots showed the differences in immune cell infiltrations between high-risk and low-risk groups. 
A

Altered in $172(99.42 \%)$ of 173 samples.

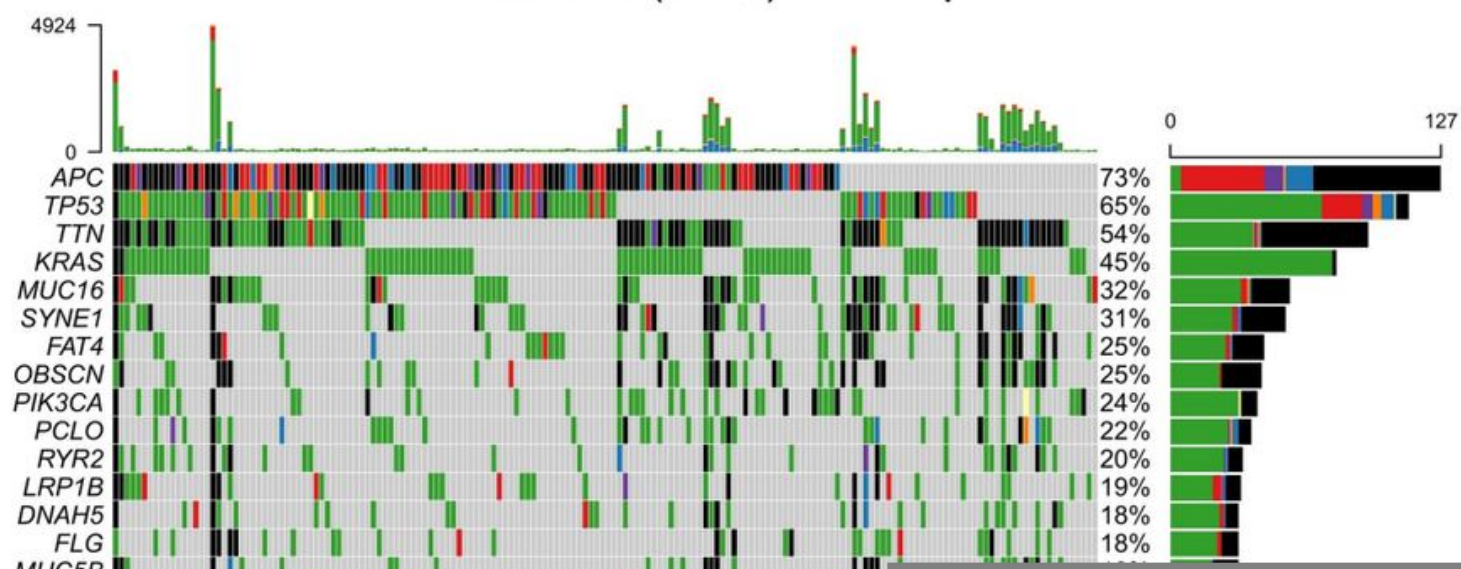

Figure 8

Mutation landscape of the prognostic signature in TCGA cohort. Waterfall plots of frequently mutated genes in (A) high-risk and (B) low-risk groups. 

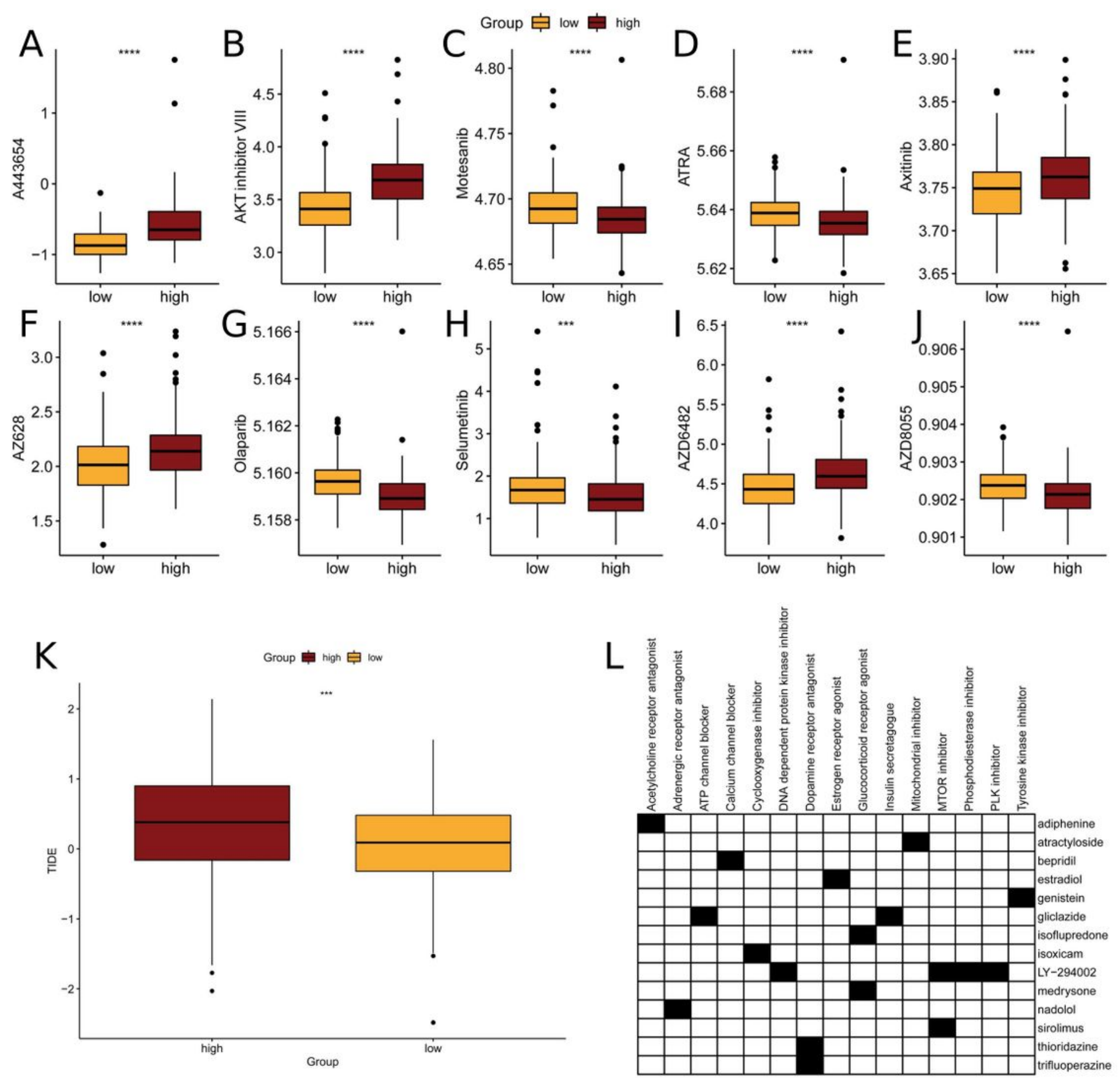

Figure 9

Differences in response to chemotherapy drug and ICB therapy among patients with different risk scores.

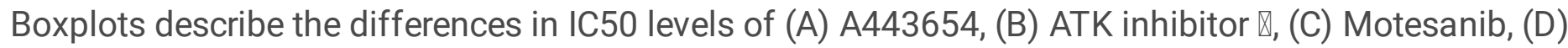
ATRA, (E) Axitinib, (F) AZ628, (G) Olaparib, (H) Selumetinib, (I) AZD6482 and (J) AZD8055 between highrisk and low-risk groups. (K) The boxplot showed the differences of TIDE scores between the two groups. (L) Heatmap showed small-molecule compounds and their drug mechanisms of action.

\section{Supplementary Files}


This is a list of supplementary files associated with this preprint. Click to download.

- SupplementaryFigure1.jpg

- SupplementaryFigurelegend.docx

- SupplementaryTable1.docx 